\title{
Avaliação e futuro do Programa Academia da Saúde
} Evaluation and future of Academia da Saúde Program

AUTHOR'S
Gregore I Mielke ${ }^{1}$ (DD
Deborah C Malta ${ }^{2}$ (D)
1. School of Human Movement and Nutrition
Sciences, The University of Queensland, Brisbane,
Queensland, Australia.
2 Programa de Pós Graduacão em Saúde Pública,
Universidade Federal de Minas Gerais, Belo
Horizonte, Minas Gerais, Brasil.
CORRE SPONDING
Gregore I Mielke
g.ivenmielke@uq.edu.au
School of Human Movement and Nutrition
Sciences (\#26B), Rm 408. The University of
Queensland | St Lucia Campus. Brisbane,
QLD 4072, Austrália.
DOI

$10.12820 /$ rbafs. $25 \mathrm{e} 0147$

\section{(cc) BY}

Este trabalho está licenciado com uma Licença Creative Commons - Atribuição 4.0 Internacional.
O Programa Academia da Saúde (PAS), o maior o maior programa de promoção da saúde já implementado no Brasil e, provavelmente no mundo, está próximo de completar a sua primeira década. Para celebrar e refletir sobre o futuro do programa, este volume especial da Revista Brasileira de Atividade Física e Saúde traz uma série de estudos que oferecem um panorama das ações de implementação, avaliação e monitoramento do PAS. Cabe destacar que a condução dos estudos de avaliação do PAS apresentados neste volume somente foi possível devido ao financiamento público de pesquisa, investimento na formação de recursos humanos e a autonomia de pesquisadores e universidades brasileiras, os quais, em conjunto com o Sistema Único de Saúde (SUS) e o Estado de bem-estar social, têm sofrido constantes ataques recentemente.

A criação do PAS, instituído nas Portarias GM/MS 719, de 07 de abril de 2011 e no 2.681, de 7 de novembro de 2013', ocorreu a partir de resultados promissores de experiências comunitárias como do Programa Academia da Cidade nas cidades de Recife, Curitiba, Vitória, Aracaju e Belo Horizonte e de outros programas similares ${ }^{2}$. Dessa forma, é fundamental destacar que a criação do PAS ocorreu num contexto de articulação política e de uma agenda que convergia para enfrentamento das doenças e agravos não transmissíveis e seus fatores de risco no âmbito SUS através de ações de promoção da saúde e redução de desigualdades, tendo a Política Nacional de Promoção da Saúde como um marco desse processo ${ }^{3-4}$.

A utilização de evidências científicas para apoiar o processo decisório de políticas públicas relacionadas ao PAS deve ser compartilhada. Dessa forma, os autores envolvidos neste volume especial sistematizaram, com rigor científico, evidências produzidas por meio de avaliações multidisciplinares, discutindo as facilidades e dificuldades vivenciadas na difícil missão de implementar e manter um dos maiores programas de promoção da saúde da história. Todos os artigos apresentados nesse volume também têm como objetivo disseminar os métodos e resultados do PAS e, dessa forma, servir como ferramentas, para gestores e profissionais municipais, estaduais e federais que lidam com o Programa, bem como para pesquisadores, comunidade acadêmica e profissionais que trabalham com o tema tanto no Brasil como no exterior.

Alguns dos resultados apresentados nesse volume corroboram com o papel fundamental que as políticas públicas têm para redução das desigualdades em saúde, particularmente quanto se trata da prática de atividade física no lazer - tema de interesse comum desta comunidade cientifica. Por exemplo, um conjunto de avaliações tem demonstrado que o perfil majoritário dos usuários do Programa é composto por grupos populacionais com menos acesso às práticas de promoção da saúde com foco em atividade física ${ }^{2,5}$. Se faz necessário reforçar que tais resultados estão alinhados com a importância das políticas 
públicas vinculadas com os princípios do SUS para que haja avanço de ações de promoção das atividades físicas de lazer contextualizadas socialmente que sejam capazes de priorizar aqueles que mais necessitam ${ }^{6}$.

Como todo programa a ser implementado num país de dimensões continentais e com mais de 5.700 municípios como é o caso do Brasil ${ }^{3}$, inúmeras fragilidades ainda precisam ser corrigidas através do controle social. Dentre elas destacamos a sustentabilidade dos processos, garantia de financiamento para novas instalações, o custeio do programa, a universalização da prática e a continuidade nos processos de capacitação, monitoramento e avaliação do programa ${ }^{2,4}$. Apesar de inúmeras dificuldades, como as sucessivas trocas de gestores, limitações dos gestores locais para condução dos processos, descontinuidade de financiamentos, os resultados das avaliações conduzidas mostram uma perspectiva na qual o 'copo está meio cheio, ${ }^{2,4}$. O PAS tem sido progressista no formato de práticas de promoção da saúde, particularmente por dialogar com as demandas locais e dos usuários do programa, resultando em um projeto promissor, que recebe apoio da gestão local, da população, e tem se se mantido ao longo das gestões ${ }^{2,4}$.

Por fim, gostaríamos que os resultados apresentados neste volume especial sobre um programa público de promoção da saúde provoquem a reflexão sobre os discursos reproduzidos rotineiramente no contexto social e político vivido no Brasil. Enquanto defensores de ações públicas de promoção da saúde ${ }^{7}$ para redução das desigualdades na prática de atividade física ${ }^{8}$, devemos estar atentos ao debate atual sobre o tamanho e o papel do Estado. É necessária a compreensão da incoerência existente no discurso que clama por políticas públicas para promoção da atividade física, quando medidas de austeridade implementadas no Brasil nos últimos anos têm sido ignoradas ou defendidas. Nesse sentido, a aprovação da Emenda Constitucional 95 (EC95) em 2016, irá afetar significativamente a oferta de ações e serviços do SUS, em especial as ações de promoção a saúde ${ }^{9}$. O congelamento orçamentário proposto com a EC95 irá impactar diretamente no Programa Academia da Saúde, assim como nos investimentos em saúde e políticas sociais, aprofundando as desigualdades e iniquidades já existentes ${ }^{9}$ e afetando ações de assistência e de promoção a saúde. É preciso estar atento!

\section{Conflito de interesse}

Os autores declaram não haver conflito de interesses.

\section{Contribuição dos autores}

Mielke GI \& Malta DC participaram na concepção, redação e revisão crítica do texto.

\section{Referências}

1. Ministério da Saúde. Portaria n. 2.681, de 7 de novembro de 2013: redefine o Programa Academia da Saúde no âmbito do Sistema Único de Saúde. 2013. [citado em 2020 out 17]. Disponível em: http://bvsms.saude.gov.br/bvs/saudelegis/ gm/2013/prt2681_07_11_2013.html.

2. Pesquisas de avaliação do Programa Academia da Saúde [recurso eletrônico] / Deborah Carvalho Malta, Grégore Iven Mielke, Nathália Cristina Pereira da Costa, organizadores. 1. ed. - Florianópolis-SC: Sociedade Brasileira de Atividade Física e Saúde, 2020. 240 p.: il.; http://sbafs.org.br/public/ Painel/midia/imagem/arquivo/Malta_Mielke_Costa_ Academia_da_Saude.pdf.

3. Ministério da Saúde. Política Nacional de Promoção da Saúde: revisão da Portaria MS/GM no 687, de 30 de março de 2006. Brasília: Ministério de Saúde, 2014.

4. Ministério da Saúde. Panorama Nacional de Implementação do Programa Academia da Saúde. Monitoramento nacional da gestão do Programa Academia da Saúde. Brasília (Brasil): Ministério da Saúde; 2017. [citado em 2020 out 17]. Disponível em: http://bvsms.saude.gov.br/bvs/publicacoes/ panorama_academia_saude_monitoramento_programa.pdf.

5. Simões EJ, Hallal PC, Siqueira FV, Schmaltz C, Menor D, Malta DC, et al. Effectiveness of a scaled up physical activity intervention in Brazil: A natural experiment. Prev Med. 2017;103(S):66-72.

6. Crochemore-Silva I, Knuth AG, Mielke GI, Loch MR. Promotion of physical activity and public policies to tackle inequalities: considerations based on the Inverse Care Law and Inverse Equity Hypothesis. Cad Saude Publica. 2020 Jun 8;36(6): 000155119.

7. Ferreira RW, Caputo EL, Häfele CA, Jerônimo JS, Florindo AA, Knuth AG, et al. Acesso aos programas públicos de atividade física no Brasil: Pesquisa Nacional de Saúde, 2013. Cad Saúde Pública 2019;35:e00008618.

8. Mielke GI, Malta DC, de Sá GB, Reis RS, Hallal PC. Regional differences and correlates of leisure time physical activity in Brazil: results from the Brazilian National Health Survey-2013. Rev Bras Epidemiol. 2015;18 Suppl 2:158-69.

9. Brasil. Emenda Constitucional no 95 , de 15 de dezembro de 2016. Altera o Ato das Disposições Constitucionais Transitórias, para instituir o Novo Regime Fiscal, e dá outras providências. Diário Oficial da União 2016; 15 dez.

Recebido: $29 / 05 / 2020$

Aprovado: 19/10/2020 